\title{
COMPLETE SETS OF REPRESENTATIONS OF ALGEBRAS
}

\section{ROBERT STEINBERG}

1. Introduction and results. A classical theorem [1, Chapter XV, Theorem IV] states:

(1) Let $G$ be a finite group and $R$ a faithful representation ${ }^{1}$ of $G$ over a field $K$. Then each irreducible representation of $G$ over $K$ is a constituent of some tensor power of $R$.

The only proof of this result known to us actually requires the additional assumption that $K$ is of characteristic 0 and involves a calculation with characters which is not very revealing (to us). In an attempt to construct a more conceptual proof we have been led to a considerably more general result.

(2) Let $A$ be an algebra over a field $K$. Assume that $A$ has a basis $B$ over $K$ such that $B \cup\{0\}$ is closed under multiplication. Finally, let $R$ be a representation of $A$ which is faithful on $B \cup\{0\}$, and for each $r=1,2, \cdots$ let $\otimes^{r} R$ be the representation of $A$ defined by $\left(\otimes^{r} R\right)(b)$ $=\otimes^{r} R(b) \quad(b \in B)$ together with linearity. Then the representations $\otimes^{r} R(r=1,2, \cdots)$ form a complete set of representations of $A$ (in the sense that their direct sum is faithful on $A$ ).

Observe that the assumptions on $B$ imply that each $\otimes^{r} R$ really is a representation of $A$ and that $A$ is associative, but that there is no restriction on the characteristic of $K$ or the dimension of $A$ or $R$. The transition from (2) to (1) is immediately effected by applying to the group algebra of $G$ the statement (2) and the following probably wellknown result, for which a proof is sketched at the end of this paper.

(3) If $\{r R \mid r=1,2, \cdots\}$ is a complete set of representations of a finite-dimensional algebra $A$, then each irreducible representation of $A$ is a constituent of some ${ }^{r} R$.

That the finiteness assumptions cannot be dropped in (1) or (3) may be seen from the following example. Let $e(k)$ be the real $2 \times 2$ matrix obtained by replacing the 12 entry of the identity matrix by $k, G$ the multiplicative group of all $e(k), A$ the group algebra of $G$ over the reals, $B$ the set $G$ (imbedded in $A$ ), and $R$ the defining representation of $G$ extended to $A$. Then no tensor power of $R$ contains the one-dimensional representation $S$ of $A$ (or $G$ ) defined by $S(e(k)$ ) $=\exp k$ ( $k$ real $)$.

The proof of (2) depends on the following lemma.

Received by the editors September 21, 1961.

1 Throughout this note all representations are assumed to correspond to left modules and the 0 -representation is excluded from the list of irreducible representations. 
(4) If $C$ is a set of nonzero elements of a vector space $V$, then in the strong direct sum $\sum_{r=1}^{\infty} \otimes^{r} V$ the vectors $\sum \otimes^{r} c(c \in C)$ are linearly independent.

2. Proofs. If the conclusion of (4) does not hold, there is a minimal nonempty finite subset $D$ of $C$ such that there are nonzero scalars $k(d)(d \in D)$ for which

$$
\sum_{d \in D} k(d) \otimes r d=0 \quad(r=1,2, \cdots) .
$$

Since $D$ clearly has at least two elements, there is a linear function $v^{*}$ on $V$ which is not constant on $D$. Replacing $r$ by $r+s$ in $\left(^{*}\right)$, taking the tensor product with $\otimes^{s} v^{*}$, and then contracting, we get

$$
\sum_{d \in D}(k(d) \otimes r d) v^{*}(d)^{s}=0 \quad(r=1,2, \cdots ; s=0,1,2, \cdots) .
$$

Thus if $k_{1}, k_{2}, \cdots, k_{n}$ are the distinct values taken by $v^{*}$ on $D$, the value $k_{1}$ being taken on the subset $D_{1}$ of $D$, then because the van der Monde matrix $\left(k_{t}^{s}\right)(1 \leqq t \leqq n, 0 \leqq s \leqq n-1)$ is nonsingular, the equations (*) hold with $D$ replaced by $D_{1}$, contradicting the minimal nature of $D$. Thus (4) is established.

Under the assumptions of (2) let $a=\sum k(b) b(b \in B, k(b) \in K)$ be an element of $A$ such that $\left(\otimes^{r} R\right)(a)=0$ for $r=1,2, \cdots$. Then $\sum k(b) \otimes r R(b)=0$ for $r=1,2, \cdots$, each $k(b)$ is 0 by (4), whence $a$ is also 0 . Thus (2) is proved.

For the proof of (3) one may assume that $\{r R\}$ is finite and consists of finite-dimensional representations. Let ${ }^{r} M={ }^{r} M_{0} \supset{ }^{r} M_{1} \supset \cdots$ be a composition series for the $A$-module ${ }^{r} M$ corresponding to ${ }^{r} R$, and let $N$ be an arbitrary irreducible $A$-module. If $A^{0}$ is the radical of $A$, then $A / A^{0}$ is a sum of minimal left ideals. Hence there is a minimal left ideal $I / A^{0}$ such that $I N \neq 0$, and then there is a corresponding pair $(r, i)$ such that $I\left({ }^{r} M_{i} /{ }^{r} M_{i+1}\right) \neq 0$, since otherwise $I$ would be nilpotent because $\{r R\}$ is complete and thus would be contained in $A^{0}$. If $m$ and $n$ are nonzero elements of ${ }^{r} M_{i} /{ }^{r} M_{i+1}$ and $N$ respectively, it is then readily verified that the map $i m \rightarrow i n(i \in I)$ is an $A$-module isomorphism of ${ }^{r} M_{i} /{ }^{r} M_{i+1}$ on $N$. Hence (3).

\section{REFERENCE}

1. W. Burnside, Theory of groups of finite order, 2nd ed., Cambridge Univ. Press, Cambridge, 1911.

University of California, Los Angeles and The Institute For Advanced Study 